\title{
Unsteady Flow free movement of Nano-fluids on permeable expanded vertical plate
}

\author{
Ehsan SUORI ${ }^{1}$, Arash Shams TALEGHANI ${ }^{2 *}$ \\ 1- Department of Mechanical Engineering, Brojerd Branch, Islamic Azad university, Brojerd, \\ Iran \\ 2- Aerospace Research Institute, Ministry of Science, Research and Technology, Tehran, Iran
}

\begin{abstract}
In this study, fluid flow and heat transfer is investigated numerically on an expanded hydrodynamic flow of Unsteady Flow in the presence of magnetic nano-fluids. In this regard, various metals will be considered as nanoparticles with water as base fluid. Nano-fluid that caused by the distribution of Nano -scale particles in conventional fluid, is a new generation of fluids with great potential in industrial applications. The effects of various physical parameters of the problem will be evaluated, such as the volume fraction of nanoparticles, different types of nanoparticles, magnetic parameters, flotation parameters, unsteady parameter, and suction on the curve of speed and temperature parameters. The results showed an increase in heat transfer due to increased volume fraction of the nanoparticles.
\end{abstract}

Keywords: Unsteady Flow, nano-fluid, heat transfer, porous vertical plate

\section{Introduction}

Increasing the heat transfer is always one of the major issues discussed in the industry. There has been a lot of attention recently on the heat transfer increment in many applications for heating and cooling, especially in small dimensions. There are many ways to increase heat transfer in heating and cooling applications. To increase the heat transfer, change the geometry, boundary conditions and an increase in thermal conductivity of the fluid can be used. The best option for researchers is to increase the thermal conductivity of the fluid because of the limitations of the current geometry and boundary conditions. For this reason, this method has been widely studied. Typical fluids that are used in heat transfer have a lower thermal conductivity, such as water, ethylene glycol and engine oil, respectively. In contrast, the metals have a higher thermal conductivity of the fluid. So, one way to increase the thermal conductivity of fluids used in the heat, is to add the metal particles in the fluid.

With the development of nanotechnology and nano-sized particles make it possible to mix those particles with fluids. Based on the description and less problems of nanoparticles, the particles

\footnotetext{
*Electronic address: Taleghani@ari.ac.ir
} 
are better compared to the particles in a micrometer and millimeter dimensions. In fact, the small size of the particles and the low volume fraction is used, solves the problems such as corrosion and pressure drop. One of the most important factors in choosing nano-fluids, is the stability of nano-particles in the fluids in the heat exchanges. Finally, the smaller particles are, however, also increases the relative level of heat transfer. As a result, the thermal efficiency of particulate matter that is a function of the heat transfer surfaces increases by reducing the particle size.

Choi and Eastman were the first people in any National Laboratory in the United States, called nano-fluids suspension of nano-particles in base fluid and showed an extraordinary increase in heat transfer coefficient [1]. Experimental results show that adding 1 to $5 \%$ by volume fraction of nano-particles can increase thermal conductivity of the mixture up to $20 \%$ compared with the base fluid [2, and 3]. This increase in heat transfer cannot be attributed exclusively to the thermal conductivity of the nanoparticles. Other mechanisms such as particle density, volume fraction, Brownian motion, particle size, shape and particle surface area also has a direct impact in heat transfer increment.

Mustafa and colleagues [4] examined the stagnation point flow on a stretching sheet of nanofluids using the homotopy analysis and taking into account the effect of Brownian motion. Steady flow of an incompressible viscous fluid on a rotating disk in the presence of nanofluids numerically using finite difference method Claire box were studied by Bachok and colleagues [5]. Aziz et al. [6] studied the natural convection flow of nanofluids on the vertical plane numerically. As expected, in all mentioned articles, the heat transfer rate with increasing volume fraction of nanoparticles will increase.

Also, Buongiorno [7] examined the phenomenon of convection heat transfer in nanofluids and found that among seven slip mechanisms, Brownian diffusion and thermophoresis will lead to a huge increase in the absolute value of the thermal conductivity of fluids. He also presented a two-component four-equation nonhomogeneous equilibrium model for mass, momentum, and heat transport in nanofluids, which involves the simultaneous effects of Brownian diffusion and thermophoresis. Nazari and colleagues [8] studied water and heat transfer fluid nano alumina / water at a constant temperature tube containing a porous material in the range of 700 to 5000 Reynolds experimentally. Mohammadpour fard [9] studied thermal and hydrodynamic behavior of Non-Newtonian ferrofluid flow through a vertical rectangular channel and in the presence of different magnetic fields numerically using a two-phase mixture model, power law model and finite volume method. Ziaee rad and Kasaei poor [10] studied similarity solution for mixedconvection copper-water nanofluid boundary layer flow over a horizontal plate using finitedifference Keller-Box method.

The purpose of the study is to investigate the fluid flow and heat transfer in nanofluids of unsteady fluid flow on a porous expanded plate in the presence of MHD flow using Runge-Kutta method. Study of heat transfer on a porous expanded plate is very vital by the wide applications in chemical engineering. Many processes in chemical engineering like as metallurgy process and polymer extrusion process involves cooling the molten liquid and drawing it into a cooling 
system. In this process, the rate of cooling and shrinking is very impressive on the quality of the final product.

\section{Statement of the problem and governing equations}

Unsteady two-dimensional incompressible laminar flow free movement of nano-fluids, has been considered in the presence of an external uniform magnetic field on a vertical permeable expanded accelerating plate. Different types of nanoparticles are considered with a water-based fluid. It should be noted that the base fluid and particles are in thermal equilibrium with each other and there is no slippage between them. Also, in $t<0$ the fluid flow and heat transfer are steady. Unsteady fluid flow and heat transfer start at $t=0$ and velocity of expanding the plate is assumed as $u_{w}(x, t)=a x /(1-c t)$ along the $\mathrm{x}$ axis. The mass transfer velocity of expanding perpendicular to the plane is considered as $v_{w}(t)$. Ambient temperature $\left(T_{\infty}\right)$ has been fixed. The wall temperature was considered greater than ambient temperature $\left(T_{w}>T_{\infty}\right)$, which is intended to represent the assisting flow. For the assisting flow, the induced tension and the thermal buoyancy help each other. The plate is spread by two different directions and equal force that is applied along the $\mathrm{X}$ axis. The effects of Brownian motion and thermal diffusion of nanoparticles is also intended in equations and viscous dissipation is ignored. Also, the physical properties of the fluid are constant. Physical geometry and coordinate system is given in Figure 1. The conservation equations of continuity, momentum and energy for unsteady incompressible laminar boundary layer flow under the influence of magnetic field in a nanofluids is as follows:

$$
\begin{aligned}
& \frac{\partial u}{\partial x}+\frac{\partial v}{\partial y}=0 \\
& \rho_{n f}\left(\frac{\partial u}{\partial t}+u \frac{\partial u}{\partial x}+v \frac{\partial u}{\partial y}\right)=\mu_{n f} \frac{\partial^{2} u}{\partial y^{2}} \\
& \pm g(\rho \beta)_{n f}\left(T-T_{\infty}\right)-\sigma B^{2} u \\
&\left(\rho c_{p}\right)_{n f}\left(\frac{\partial T}{\partial t}+u \frac{\partial T}{\partial x}+v \frac{\partial T}{\partial y}\right)= \\
& k_{n f} \frac{\partial^{2} T}{\partial y^{2}}
\end{aligned}
$$

In the above equations $u$ and $v$ are velocity components along with $x, y$ and $\sigma_{\text {is }}$ the electrical conductivity, uniform magnetic field $B=B_{0} / \sqrt{1-c t}$, the specific heat $c_{p}$ at constant pressure, thermal conductivity $k, g$ is the acceleration of gravity and $T$ is an important loss of fluid temperature. In the above equations, and dynamic viscosity and density of nanofluids, which are presented by Brinkman [11] below as well as $k_{n f}$ is the effective thermal conductivity of 
nanofluids [12], $\beta_{n f}$ is thermal expansion coefficient and $\left(\rho c_{p}\right)_{n f}$ is thermal capacity of nanofluids:

$$
\begin{aligned}
& (\rho \beta)_{n f}=(1-\varphi)(\rho \beta)_{f}+\varphi(\rho \beta)_{s}, \\
& \left(\rho c_{p}\right)_{n f}=(1-\varphi)\left(\rho c_{p}\right)_{f}+\varphi\left(\rho c_{p}\right)_{s}, \\
& \frac{k_{n f}}{k_{f}}=\frac{\left(k_{s}+2 k_{f}\right)-2 \varphi\left(k_{f}-k_{s}\right)}{\left(k_{s}+2 k_{f}\right)+\varphi\left(k_{f}-k_{s}\right)}, \\
& \rho_{n f}=(1-\varphi) \rho_{f}+\varphi \rho_{s}, \\
& \mu_{n f}=\frac{\mu_{f}}{(1-\varphi)^{2.5}}
\end{aligned}
$$

Where $\mu_{f}$ is the dynamic viscosity of the base fluid, $\varphi$ is the solid volume fraction of nanoparticles, $\rho_{f}$ and $\rho_{s}$, are the density of the liquid fraction and solid fraction, respectively. It should be noted that the above equation is to calculate the spherical nanoparticles and is not used for other geometries nanofluids relationship and the relationship will change in other nanoparticles shapes. Thermo-physical properties of the base fluid (water) and various particles are given in Table 1 [13].

Table 1: thermo-physical properties of the base fluid and different particles [13].

\begin{tabular}{rrrrr}
\hline $\begin{array}{r}\text { Thermal } \\
\text { Expansion }\end{array}$ & $\begin{array}{r}\text { Thermal } \\
\text { conductivity }\end{array}$ & Density & $\begin{array}{r}\text { Specific heat at constant } \\
\text { pressure }\end{array}$ & $\begin{array}{r}\text { Physical } \\
\text { properties }\end{array}$ \\
\hline 21 & 0.613 & 997.1 & 4179 & water \\
1.67 & 400 & 8933 & 385 & $\mathrm{Cu}$ \\
0.85 & 40 & 397 & 765 & $\mathrm{Al}_{2} \mathrm{O}_{3}$ \\
0.9 & 8.9538 & 425 & 686.2 & $\mathrm{TiO}_{2}$ \\
\hline
\end{tabular}


Bulletin de la Société Royale des Sciences de Liège, Vol. : 85, 2016, p. 34 - 47

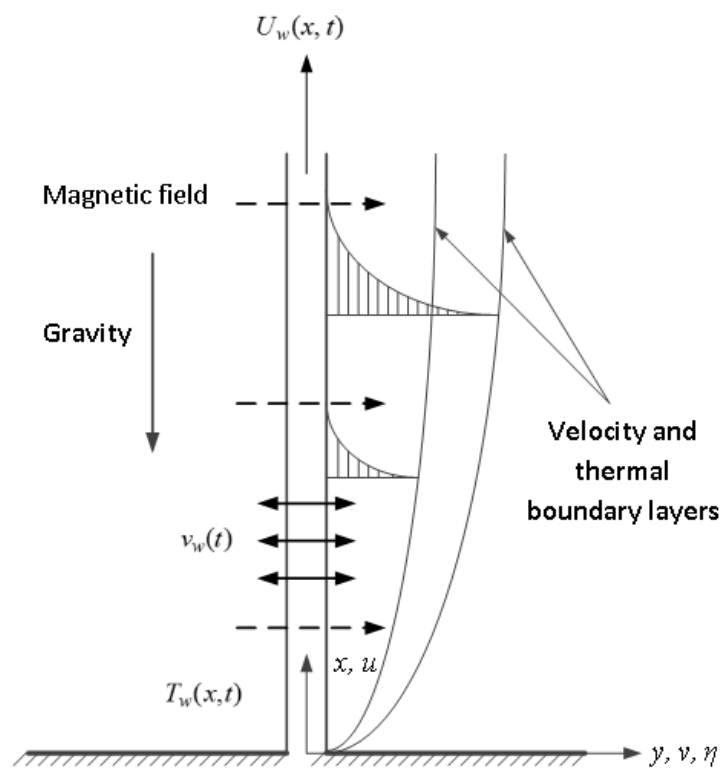

Fig.1: The current configuration on page spread and coordinates the issue.

Boundary conditions appropriate to issue the uniform suction of the page are as follows:

$$
\begin{aligned}
& u=U_{w}(x, t)=\frac{a x}{(1-c t)}, \\
& v=v_{w}(t)=\frac{v_{0}}{\sqrt{1-c t}}, \quad \text { at } y=0, \\
& T=T_{w}(x, t) \\
& =T_{\infty}+\frac{b x}{(1-c t)^{2}},
\end{aligned}
$$

$u \rightarrow 0, \quad T \rightarrow T_{\infty}, \quad$ as $\quad y \rightarrow \infty$,

In the above equations, $a$ and $c$ are constants. $a$ parameter is the initial stretch rate that increases with increasing the time $(a /(1-c t)) . b$ is also consistent with the temperature / length that $(\mathrm{b}=$ 0 ) refers to the absence of buoyancy forces. By introducing the stream function $\psi(x, y)$ as follows , continuity equation will be satisfied:

$$
\begin{aligned}
& u=\frac{\partial \psi}{\partial y}=\frac{a x}{1-c t} f^{\prime}(\eta), \\
& v=-\frac{\partial \psi}{\partial x}=-\left(\frac{v_{f} a}{1-c t}\right)^{1 / 2} f(\eta),
\end{aligned}
$$


The similarity parameter of $\eta$, dimensionless component of temperature and stream function are defined as follows:

$$
\begin{aligned}
& \eta=\left(\frac{a}{v_{f}(1-c t)}\right)^{1 / 2} y, \theta(\eta)=\frac{T-T_{\infty}}{T_{w}-T_{\infty}}, \\
& \psi(x, y)=\left(\frac{v_{f} a}{1-c t}\right)^{1 / 2} x f(\eta),
\end{aligned}
$$

Using equation (4) as well as the above mentioned similarity parameter and dimensionless component of temperature and stream function and also replacing them in equation (2) and (3), the following system of ordinary differential equations is:

$$
\begin{aligned}
& \frac{1}{(1-\varphi)^{2.5}} f^{\prime \prime \prime}(\eta)-M f^{\prime}(\eta)- \\
& \left(1-\varphi+\varphi\left(\rho_{s} / \rho_{f}\right)\right) \\
& \times\left\{\begin{array}{l}
f^{\prime 2}(\eta)-f(\eta) f^{\prime \prime}(\eta)+ \\
A\left(f^{\prime}(\eta)+\frac{1}{2} \eta f^{\prime \prime}(\eta)\right)
\end{array}\right\} \\
& +\left\{1-\varphi+\varphi \frac{(\rho \beta)_{s}}{(\rho \beta)_{f}}\right\} \lambda \theta(\eta)=0, \\
& \frac{1}{\operatorname{Pr}} \frac{k_{n f} / k_{f}}{1-\varphi+\varphi\left(\left(\rho c_{p}\right)_{s} /\left(\rho c_{p}\right)_{f}\right)} \theta^{\prime \prime}(\eta) \\
& +\left|\begin{array}{ll}
f(\eta) & \theta(\eta) \\
f^{\prime}(\eta) & \theta^{\prime}(\eta)
\end{array}\right| \\
& -A\left(2 \theta(\eta)+\frac{1}{2} \eta \theta^{\prime}(\eta)\right)=0,
\end{aligned}
$$

In the above equations, $A=c / a$ is unsteadiness parameter, $M=\sigma B_{0}^{2} / a \rho_{f}$ is magnetic parameter, $\lambda=g \beta_{f} b / a^{2}$ is buoyancy parameter, and $\operatorname{Pr}=v_{f} / \alpha_{f}$ is Prandtl number. The boundary conditions are as follows: 


$$
\left.\begin{array}{l}
\left.\begin{array}{l}
f(\eta)=f_{w}, \\
f^{\prime}(\eta)=1, \\
\theta(\eta)=1,
\end{array}\right\} \text { at } \quad \eta=0, \\
f^{\prime}(\eta) \rightarrow 0, \\
\theta(\eta) \rightarrow 0,
\end{array}\right\} \text { as } \quad \eta \rightarrow \infty,
$$

Where in the above equation $f_{w}=-v_{0} / \sqrt{v_{f} a}$ is the suction parameter.

\section{Discussion and Results}

Ordinary nonlinear differential equations (8) and (9) according to the boundary conditions (10) are solved numerically using Runge-Kutta fourth order method for different values of magnetic parameters, the volume fraction of nanoparticles, buoyancy, unsteadiness, and suction. In this section, three types of nanoparticles intended that include copper $\mathrm{Cu}$, aluminum oxide $\mathrm{Al}_{2} \mathrm{O}_{3}$ and titanium dioxide $\mathrm{TiO}_{2}$. Water is considered as a base fluid. It should be noted that copper $\mathrm{Cu}$ is considered in all forms as nanoparticles, except the forms that exist to compare the types of nanoparticles. Prandtl number is equal to 6.2 that represents the Prandtl number of water at $20{ }^{\circ} \mathrm{C}$ [13]. Also, the volume fraction of nanoparticles will be changed from zero that indicates the regular Newtonian fluid up to 0.2. First, a comparison between some of the results in this paper and previously published papers [14-16] has been done in Table 2 that show good agreement between the results.

Figures 2 and 3, investigate the effect of the volume fraction of nanoparticles on velocity and temperature distribution curves. The results show that the velocity component decreases with increasing volume fraction of nanoparticles. This phenomenon occurs because presence of the nanoparticles leads to further thinning of boundary layer thickness. On the other hand, with increasing volume fraction of nanoparticles, thermal conductivity and consequently thermal boundary layer thickness increase.

Unsteadiness parameter influence on the temperature and velocity distributions in reducing mode $(A<0)$ are shown in Figures 4 and 5. Velocity distribution increases for larger quantities of unsteadiness parameter. It means that the boundary layer thickness for larger values of absolute value of A becomes thicker. It should be noted that this behavior is reversed away from the surface. Also, the temperature distribution is increased by reducing the amount of acceleration.

Figures 6 and 7 show magnetic parameter effects on velocity and temperature profiles. A force like as drag force, that called Lorentz force generate due to the magnetic field applied perpendicularly to the fluid flow with electrical conductivity. This force tends to decrement of flow velocity near the plate along with low amount of temperature increment. Therefore, the amount of velocity decreases and thermal boundary layer thickness increases with magnetic 
parameter increment. It should be noted that, a large resistance on the fluid particles movement is caused by increasing the magnetic parameter that generate heat in the fluid.

The effects of buoyancy on velocity and temperature profiles of the fluid flow have been shown in Figures 8 and 9. The buoyancy parameter shows the amount of floating effect on heat transfer and fluid flow pattern in comparison with inertia of external forces or free stream. The results show that as buoyancy parameter increases the velocity profile also slightly increases, while the temperature distribution decreases slowly. Due to buoyancy parameter definition (the ratio of buoyancy forces to inertia forces in buoyancy layer), increment of the buoyancy parameter lead to increment of fluid velocity. In other words, since the equations are connected to each other only via the buoyancy parameters, increment of Grashof number will cause an increment in fluid velocity and thereby increment of the velocity and boundary layer thickness. The buoyant force acts like a favorable pressure gradient and accelerate to the fluid, so the boundary layer thickness increases as Grashof number increases.

Figures 10 and 11 show the suction parameter on the velocity and temperature profiles. When the suction is applied to the wall, velocity is reduced, because an amount of the fluid in suction is pulled into the walls, and consequently the boundary layer will be thinner. Gradual reduction in the thermal boundary layer thickness can be seen for large-suction parameters.

Figures 12 and 13 show the numerical results of surface friction coefficient $C_{f} \operatorname{Re}_{x}^{1 / 2}$ and the local Nusselt number $N u_{x} R e_{x}^{-1 / 2}$ for a wide range of nano-particles volume fraction parameter with three types of nano-particles. As the results show, with increasing volume fraction of nanoparticles, the absolute value of the surface friction coefficient will decrease linearly. The most amount of surface friction coefficient is related to copper nano-particles, because the copper has the highest density between the intended nano-particles. Aluminum oxide is in contrast with copper.

Table 2: Comparison of the results of compared rate of transfer- $\theta^{\prime}(0)$ for different values of Prandtl number $(\mathrm{Pr})$ on the transmission rate $M=A=\varphi=\lambda=f_{w}=0$

\begin{tabular}{ccccc}
\hline Current study & Ali[16] & Mahdi[15] & Ishak et al.[14] & $P r$ \\
\hline 0.80863 & 0.8058 & 0.80868 & 0.8086 & 0.72 \\
1.00000 & 0.9961 & 1.00000 & 1.0000 & 1 \\
1.92368 & 1.9144 & 1.92368 & 1.9237 & 3 \\
3.07225 & - & 3.07224 & 3.0723 & 7 \\
\hline
\end{tabular}


Bulletin de la Société Royale des Sciences de Liège, Vol. : 85, 2016, p. 34 - 47

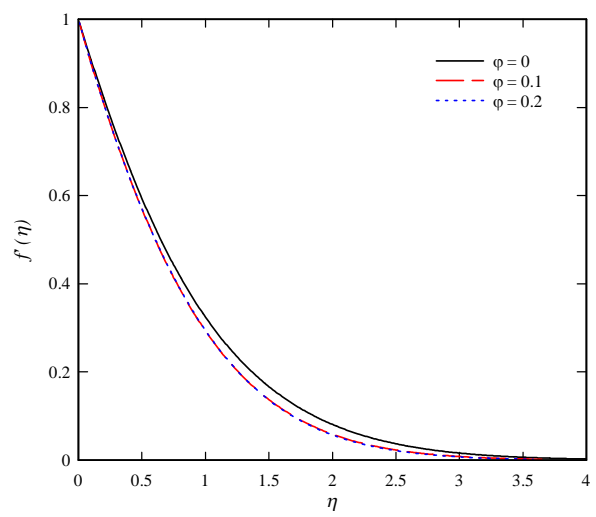

Fig. 2: The effect of the nano-particles volume fraction over velocity profile for. $-A=M=\lambda=0.5$

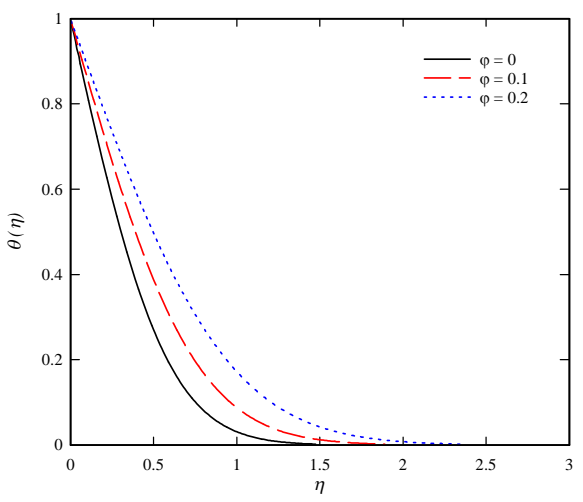

Fig. 3: The effect of the nano-particles volume fraction over temperature profile for. $-A=M=\lambda=0.5$

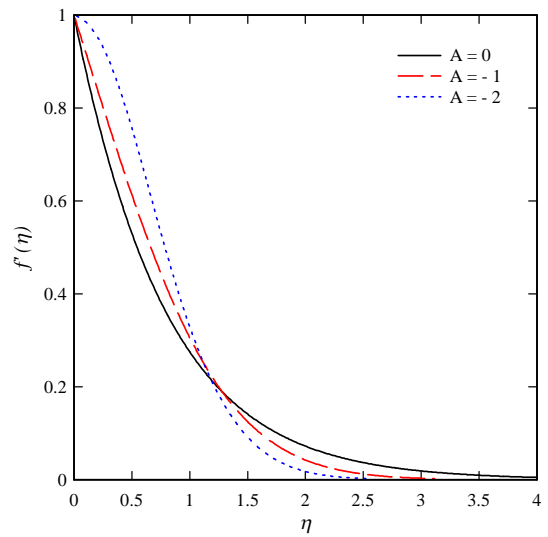

Fig. 4: The effect of the unsteadiness over velocity profile for $-A=\lambda=0.5$ and $\varphi=0.1$ 
Bulletin de la Société Royale des Sciences de Liège, Vol. : 85, 2016, p. 34 - 47

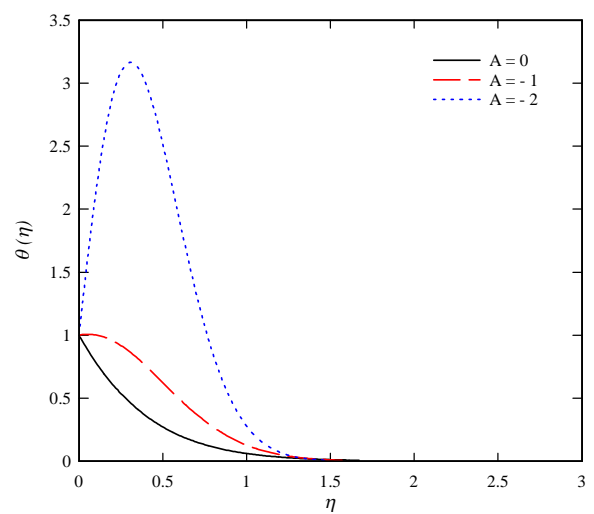

Fig. 5: The effect of the unsteadiness over temperature profile for $M=\lambda=0.5$ and $\varphi=0.1$

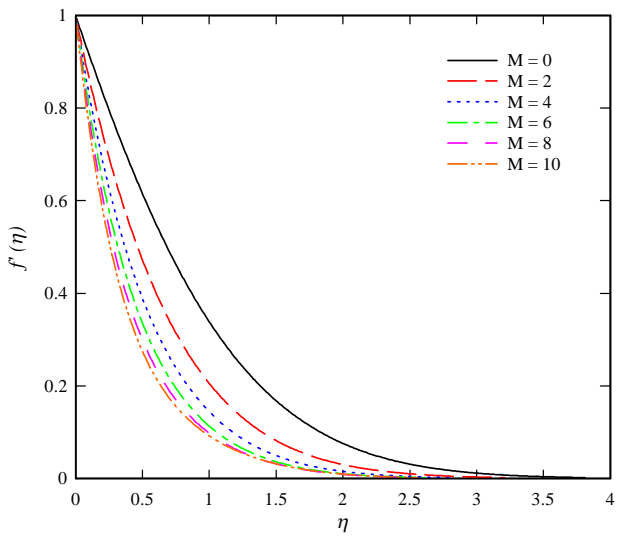

Fig. 6: The effect of the magnetic parameters on velocity profile for $M=\lambda=0.5$ and $\varphi=0.1$

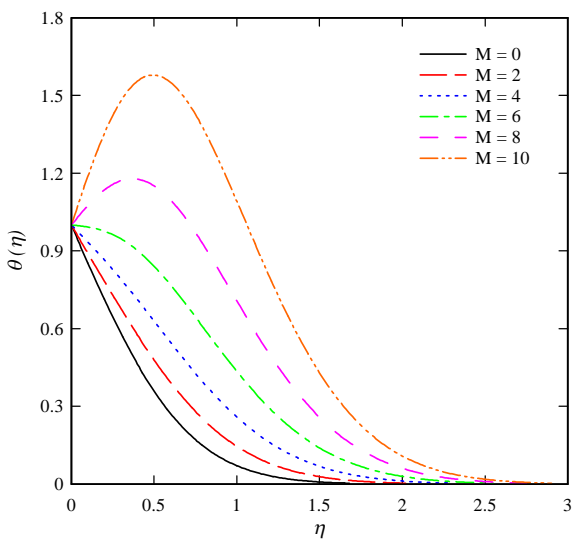

Fig. 7: The effect of the magnetic parameter on temperature profile for $M=\lambda=0.5$ and $\varphi=0.1$ 
Bulletin de la Société Royale des Sciences de Liège, Vol. : 85, 2016, p. 34 - 47

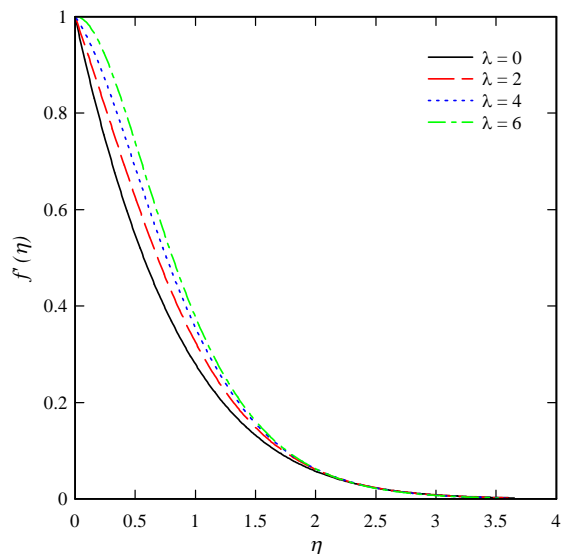

Fig. 8: The effect of the buoyancy on velocity profile for $M=\lambda=0.5$ and $\varphi=0.1$

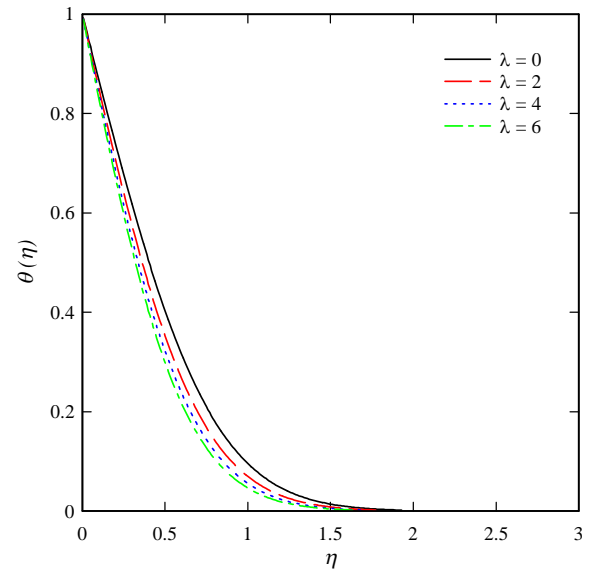

Fig. 9: The effect of the buoyancy parameter over temperature profile for $M=\lambda=0.5$ and $\varphi=0.1$

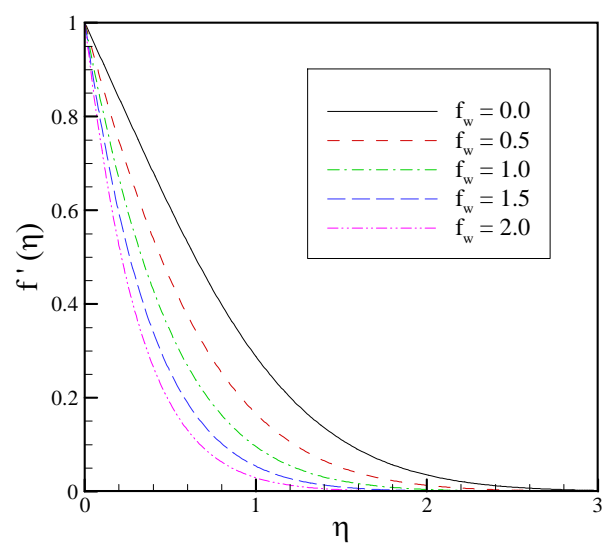

Fig. 10: The effect of the suction parameter on velocity profile for $-A=M=\lambda=1$ and $\varphi=0.1$ 
Bulletin de la Société Royale des Sciences de Liège, Vol. : 85, 2016, p. 34 - 47

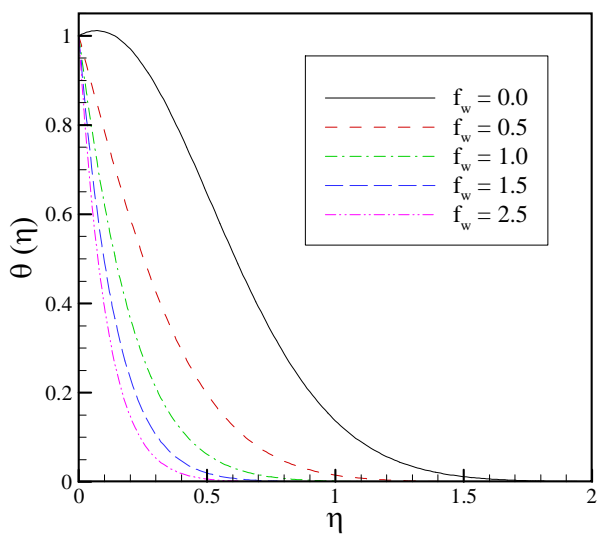

Fig. 11: The effect of the suction parameter on temperature profile for $-A=M=\lambda=1$ and $\varphi=0.1$

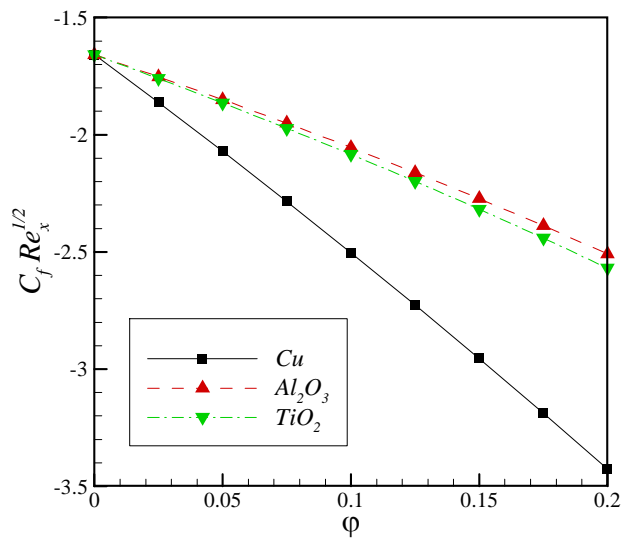

Fig. 12: The effect of the variation of nano-particles volume fraction on the surface friction coefficient at different types of nano-particles for $-A=M=\lambda=f_{w}=1$

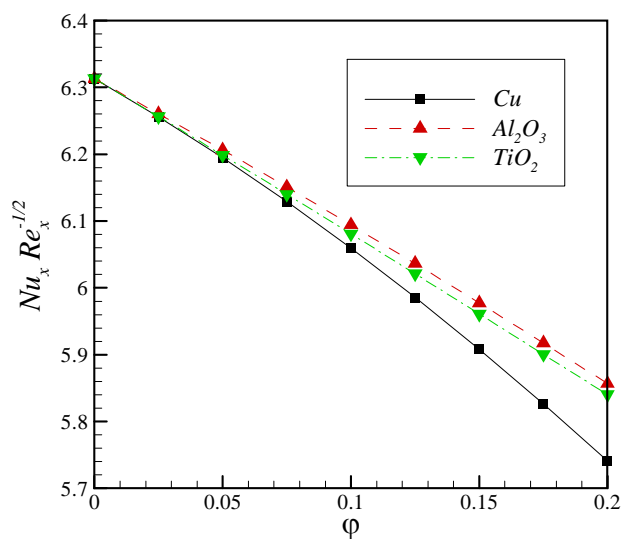

Fig. 13: The effect of the variation of nano-particles volume fraction on the Nusselt number according to different types of nanoparticles for $-A=M=\lambda=f_{w}=1$ 


\section{Conclusion}

In this study, fluid flow and heat transfer in nanofluids on the pages has been developed in the presence of magnetic hydrodynamic unsteady flow. Conservation equations in the form of partial differential equations which are solved in order to convert them into ordinary differential equations are used for the components of the velocity and temperature distribution and concentration of the zero-dimensional similarity. Dimensionless ordinary differential equations obtained numerically using Runge-Kutta and delivery techniques have been resolved. The results obtained in this study, other references and also used analytical solutions, which well be seen that shows the accuracy of the solution is in this article.

\section{References}

[1]S. U. S. Choi, J. A. Eastman, Enhancing thermal conductivity of fluids with nanoparticles, Materials Science 231 (1995) 99-105.

[2]J. A. Eastman, U. S. Choi, S. Li, G. Soyez, L. J. Thompson, R. J. DiMelfi, Novel Thermal Properties of Nanostructured Materials, Materials Science Forum 312 - 314 (1999) 629-634.

[3]Y. Xuan, W. Roetzel, Conceptions for heat transfer correlation of nanofluids, International Journal of Heat and Mass Transfer 43 (2000) 3701-3707.

[4]M. Mustafa, T. Hayat, I. Pop, S. Asghar ,S. Obaidat, Stagnation-point flow of a nanofluid towards a stretching sheet, International Journal of Heat and Mass Transfer 54 (2011) 55885594.

[5]N. Bachok, A. Ishak, I. Pop, Flow and heat transfer over a rotating porous disk in a nanofluid, Physica B: Condensed Matter 406 (2011) 1767-1772.

[6]A. Aziz, W. A. Khan, Natural convective boundary layer flow of a nanofluid past a convectively heated vertical plate, International Journal of Thermal Sciences 52 (2012) 83-90.

[7]J. Buongiorno, Convective Transport in Nanofluids, Journal of Heat Transfer 128 (2005) 240250.

[8]M. Nazari, M. Ashouri, M. H. Kayhani, Experimental investigation of forced convection of nanofluids in a horizontal tube filled with porous medium, Modares Mechanical Engineering 14 (2014)109-116.

[9]M. Mohammadpourfard, Numerical study of magnetic fields effects on the electrical conducting non-Newtonian ferrofluid flow through a vertical channel, Modares Mechanical Engineering 15 (2015) 379-389.

[10]M. Ziaei-Rad, A. Kasaeipoor, A Numerical study of similarity solution for mixed-convection copper-water nanofluid boundary layer flow over a horizontal plate, Modares Mechanical Engineering 14 (2015) 190-198.

[11]H. C. Brinkman, The Viscosity of Concentrated Suspensions and Solutions ,The Journal of Chemical Physics 20 (1952) 571-571. 
[12]H. F. Oztop, E. Abu-Nada, Numerical study of natural convection in partially heated rectangular enclosures filled with nanofluids, International Journal of Heat and Fluid Flow 29 (2008) 1326-1336.

[13]H. Oztop, E. Abu-Nada, Numerical study of natural convection in partially heated rectangular enclosures filled with nanofluids, International Journal of Heat and Fluid Flow 29 (2008) 1326-1336.

[14]A. Ishak, R. Nazar, I. Pop, Boundary layer flow and heat transfer over an unsteady stretching vertical surface, Meccanica 44 (2009) 369-375.

[15]A. Mahdy, Unsteady mixed convection boundary layer flow and heat transfer of nanofluids due to stretching sheet, Nuclear Engineering and Design 249 (2012) 248-255.

[16]M. E. Ali, Heat transfer characteristics of a continuous stretching surface, Wärme - und Stoffübertragung 29 (1994) 227-234. 